\title{
Comparação entre valores espirométricos de referência obtidos a partir das equações de Knudson e de Pereira - Adultos*
}

\author{
Waldemar Ladosky ${ }^{1}$, Rogerson T. Andrade ${ }^{2}$, Noel Guedes Loureiro ${ }^{2}$, Jesus M.B. GandaR ${ }^{2}$, Marcos M. BotelhO ${ }^{3}$
}

Em revisão de 1991, a American Thoracic Society (ATS) recomenda que cada região defina equações próprias para obtenção dos valores teóricos (VTs) dos parâmetros espirométricos, que traduzam a realidade funcional para a população local. Quando dessa recomendação, os EUA já dispunham de diversos sistemas de equações próprios, bem como a Comunidade Européia e a Polônia em particular. Em 1992, Pereira et al., analisando 4.200 espirogramas de brasileiros normais, encontraram VT para capacidade vital forçada (CVF), volume expiratório forçado de 1 seg. $\left(\mathrm{VEF}_{1}\right)$ e de fluxo expiratório médio

$\left(\mathrm{FEF}_{25-75}\right)$ diferentes dos descritos pelos autores americanos e europeus. Este estudo foi realizado para comparar as equações de Pereira et al., desenvolvida a partir de amostra de população brasileira, e as americanas de Knudson et al., bastante utilizadas em nosso meio. Foi também investigada a conseqüente influência nos laudos espirométricos, quando uma ou outra é empregada. Foram analisados 1.070 espirogramas de pacientes de ambos os sexos (389 homens e 681 mulheres) que vieram ao serviço para avaliação funcional de rotina. Não foi excluída qualquer patologia nem levada em conta a situação tabágica do paciente. Para cada paciente foi feito o cálculo do VT para CVF, $\mathrm{VEF}_{1} e$ $\mathrm{FEF}_{25.75}$ segundo as equações de Pereira (VT-P) e de Knudson 1983 (VT-K) e comparados as médias, desvios padróes e os laudos obtidos por um e por outro. Os VT-K para a CVF foram de $4,01 \%$ [ $p<0,005$ ] maiores entre as mulheres, levando a sobrevalorização das restrições respiratórias, mas entre os homens não houve diferença relevante. Quanto à $\mathrm{VEF}_{1}$, OS VT-P foram superiores tanto em homens $(4,76 \%$ [p < 0,005]) como em mulheres $(5,04 \%$ [p < 0,05]), levando a maior sensibilidade na identificação de obstruções respiratórias. Não se observou diferença relevante entre os VT-P e VT-K para o $\mathrm{FEF}_{25-75}$ em nenhum dos sexos. (J Pneumol 2001;27(6):315-320)

\section{Comparing reference spirometric values obtained from Knudson and Pereira equations - Adults}

In its 1991revision, the American Thorax Society (ATS) recommended that each region should define its own equations to determine the theoretical, or predicted values of the spirometric parameters that best represent the pulmonary functional reality of the local population. At that time, the United States already had various equations of its own as well as the European

Community, specially Poland. In 1992, Pereira et al., analysing 4,200 spirograms on healthy Brazilians, found predicted values (PV) of forced vital capacity (FVC), forced expiratory volume in 1 $\sec \left(\mathrm{FEV}_{1}\right)$ and middle expiratory flow (MEF) different from those reported by American and European authors. The purpose of the present study was to compare the equations developed by Pereira et al., using a Brazilian sample population, with those determined by Knudson et al. for American populations, that are the most used by Brazilian specialists. This study included 1,070 spirograms performed in patients of both genders (389 males and 681 females) who came to our service for routine functional assessment. No pathology was excluded and the smoking status of

the patients was not taken into account. For each patient the PV of FVC, FEV ${ }_{1}$ and MEF were calculated accordingly to the equations of Pereira et al. (PV-P) and Knudsen et al. (PV-K).

Subsequently, the means, standard deviations and reports obtained by both methods were compared. Among females, the PV-K of FVC were $4,01 \%$ ( $p<0.005)$ greater, leading to a higher

\footnotetext{
* Trabalho realizado no Laboratório de Função Pulmonar do Hospital das Clínicas da Universidade Federal de Pernambuco, PE.

1. Professor Titular.

2. Estudante de Medicina.

3. Professor Adjunto.
}

Endereço para correspondência - Av. Prof. Morais Rego, s/n, Cidade Universitária - 50670-420 - Recife, PE. Fax (81) 271-8534; E-mail: doskyla@hotlink.com.br

Recebido para publicação em 30/5/01. Aprovado, após revisão, em 17/9/01. 
rate of restrictive impairment whereas among males there was no significant difference.

Regarding $\mathrm{FEV}_{1}$, PV-P were higher in both males $(4.76 \% ; \mathrm{p}<0.005)$ and females $(5.04 \% ; \mathrm{p}<0.05)$ resulting in a greater sensitivity in the identification of respiratory obstructions. No significant difference was observed between PV-P and $\mathrm{PV}-\mathrm{K}$ as to $\mathrm{FEF}_{25-75}$ regardless of gender.

Descritores - Espirometria. Valores de referência. Fluxo expiratório forçado.

Key words - Spirometry. Reference values. Forced expiratory flow rates.

\section{INTRODUÇÃO}

Os principais parâmetros espirométricos $\left(\mathrm{CVF}, \mathrm{VEF}_{1}, \mathrm{FE}_{\max }\right.$ e $\mathrm{FEF}_{25-75}$ ) apresentam dimorfismo sexual(1-5). Variam também segundo a origem étnica do paciente $e^{(4,6-8)}$, seguem flutuação etária ${ }^{(1-3,9-13)}$ e dependem da altura ${ }^{(3)}$. Desde o trabalho pioneiro de Hutchinson, em $1846^{(14)}$, reconhece-se que as medidas espirométricas somente podem ser analisadas em comparação com valores obtidos para uma população padrão, aos quais se denomina valor de referência ou valor teórico (VT) ${ }^{(2,15-16)}$.

Nos Estados Unidos diversas propostas de equações para cálculo do VT foram descritas, sendo as mais utilizadas as de Knudson et al..$^{(4,10,11)}$, Crapo et al. ${ }^{(17)} e$ as de Morris et al. ${ }^{(18)}$. A Comissão Européia do Carvão e Aço (CECA) reuniu um grupo de especialistas, que estabeleceu VTs a serem adotados na Comunidade Européia ${ }^{(19)}$, diferentes dos americanos. Na Polônia foram descritas equações ${ }^{(20,21)}$ que são distintas das da CECA e a Austrália e Nova Zelândia ${ }^{(22)}$ utilizam equações próprias.

As diferenças entre equações de VT são tão importantes que a American Thorax Society (ATS), em revisão de normas de $1991^{(23)}$, recomenda que para cada grupamento populacional sejam escolhidas equações próprias que mais se adaptem à sua realidade. Já em 1978, Romaldi$\mathrm{ni}^{(24)}$ havia salientado a necessidade de padronizar uma equação brasileira, o que foi feito por Pereira et al. ${ }^{(25)}$, em 1992.

Talvez devido ao fato de que os espirômetros importados, de uso mais corrente, já trazem consigo as equações de VT do país de origem, estas têm sido preferencialmente utilizadas entre nós, sendo as de Knudson ${ }^{(10-12)}$ as mais empregadas, especialmente esta última, publicada em 1983.

O presente estudo propõe-se comparar os VTs obtidos para CVF, $\mathrm{VEF}_{1}$ e $\mathrm{FEF}_{25-75}$, a partir das equações de Knudson et al. de $1983^{(12)}$ e de Pereira et al. ${ }^{(25)}$ e qual sua influência nos laudos espirométricos resultantes.

\section{MATERIAL E MÉTODOS}

\section{Pacientes}

Foram analisados 1.070 pacientes de ambos os sexos (389 masculinos e 681 femininos), com idade entre 17 e
60 anos, que vieram de rotina ao Serviço, enviados para avaliação da função pulmonar pelos ambulatórios de Pneumologia, Reumatologia e Cirurgia Geral. Embora pacientes entre 16 e 17 anos com estatura igual ou superior a $170 \mathrm{~cm}$ tenham seus VTs calculados pelas equações de adultos, em nossa amostra populacional nenhum se enquadrou nesses critérios.

Os pacientes foram divididos em faixas etárias correspondentes às décadas de vida. Apenas a primeira faixa etária foi um pouco mais alargada para englobar os poucos pacientes com menos de 20 anos de idade.

Analisando a regressão $\mathrm{VEF}_{1}$ /estatura versus idade, Pereira et al. ${ }^{(25)}$ observaram que a inclinação se tornava negativa a partir dos 25 anos nos indivíduos do sexo masculino, da mesma forma que Knudson et al. ${ }^{(12)}$; e aos 20 anos no sexo feminino, sendo estas então consideradas como o início da idade adulta para a função respiratória.

Como há, entretanto, discrepâncias ${ }^{(26-28)}$ quanto à idade na qual a função pulmonar atinge seu ápice de desenvolvimento passando em seguida a decrescer, foram tomados como base os dados de Dickman et al. ${ }^{(26)}$ e de Seely et al. ${ }^{(27)}$, que referem a estabilização da função respiratória aos 18 anos.

Como as equações propostas para adolescentes brasileiros $^{(29)}$ chegam apenas à idade de $17-18$ anos para indivíduos com alturas inferiores a $170 \mathrm{~cm}$, fica um intervalo, sem valor teórico definido, entre esta idade e as definidas por Pereira et al. ${ }^{(25)}$.

Por essa razão, os pacientes da $1^{\text {a }}$ faixa etária foram divididos em dois subgrupos: um extrapolado para a faixa etária entre 17 e 24 anos para o sexo masculino e 17 e 20 anos para o feminino, e outro iniciando na idade proposta por Pereira et al. ${ }^{(25)}$ como o início da idade adulta para a função respiratória. A influência da extrapolação é discutida na análise dos resultados.

Não foram levados em conta os diagnósticos prévios dos pacientes, nem o fato de serem ou não tabagistas.

\section{Espirogramas}

Para cada paciente foi realizada prova de espirometria forçada dentro das normas previstas pelo Consenso Brasileiro de Espirometria ${ }^{(30)}$, sendo a manobra realizada segundo D'Angelo et al. ${ }^{(31)}$. Não foram incluídos no estudo os espirogramas que apresentaram um volume extrapolado $>5 \%$ da CVF, ou que possuíssem indícios de má execução, como finalização precoce, fechamento de glote, tosse. 
Para cada espirograma foi feito o cálculo do valor teórico segundo as equações de Knudson (VT-K) e de Pereira (VT-P) para CVF, $\mathrm{VEF}_{1}$ e $\mathrm{FEF}_{25-75}$ e os resultados comparados. O valor espirométrico, obtido para cada um dos parâmetros em estudo, foi avaliado em relação a cada valor teórico. Para cada equação (Pereira; Knudson) avaliando um mesmo espirograma foi estabelecido um laudo, segundo o Consenso Brasileiro de Espirometria ${ }^{(30)}$.

As provas eram realizadas invariavelmente pela manhã com a sala mantida a $25^{\circ} \mathrm{C}$, com o paciente sentado. Ao paciente era solicitado a realizar pelo menos três manobras, sendo selecionada a que apresentasse melhor soma de $\mathrm{CVF}+\mathrm{VEF}_{1}$, e em que esses parâmetros não diferissem entre si em mais de $100 \mathrm{ml}$ ou $5 \%$.

Foi tomado o quinto percentil como limite para normalidade, abaixo do qual foram estabelecidos os diagnósticos de doença obstrutiva, restritiva ou mista, conforme o caso.

\section{Equipamento}

Foi utilizado um espirômetro fluxométrico Beatrice da EBEM (Recife-Brasil), que utiliza um pneumotacômetro Fleisch IV acoplado a um transdutor de pressão diferencial e que atende às normas da SBPT, ATS e CECA. O aparelho era calibrado toda manhã utilizando uma seringa de três litros, fazendo-se três fluxos distintos, ajustando-se para umidade, temperatura e pressão atmosférica.

O programa utilizado (Pulmosoft 4.0 - 1998) permite, por meio de seleção em menu, o cálculo automático, para cada espirometria, do valor teórico para o paciente, tanto segundo as equações de Knudson et al. ${ }^{(10)}$ como as de Pereira et al. ${ }^{(25)}$.

\section{Estatística}

Foram inicialmente calculadas as médias aritméticas e os desvios padrões para os VTs de cada parâmetro obtidos segundo Pereira e Knudson. Em seguida, foi feita a medida da diferença entre as médias dos valores teóricos correspondentes (Pereira e Knudson). Procedeu-se, então, à comparação estatística, pelo t de Student, entre as médias de cada parâmetro.

Tomando-se como padrão os laudos obtidos com a equação de Pereira, por ter sido esta desenvolvida a partir de população brasileira, foram analisadas as coincidências e divergências de laudo para cada paciente e os resultados tabelados. Foi considerado como limite de significância $\mathrm{p}<0,05$.

\section{REsultados}

Os VTs de $\mathrm{FEF}_{25-75}$ calculados pelas equações de Pereira et al. ${ }^{(25)}$ e de Knudson et al. ${ }^{(10)}$ foram idênticos entre si, independentemente de sexo ou faixa etária. Por esse motivo não serão abordados.
O diagnóstico de distúrbio respiratório misto dificilmente pode ser firmado apenas com o laudo espirométrico. Por essa razão, na análise dos laudos não serão abordados os pacientes espirometricamente classificados como "mistos".

\section{Diferenças entre as médias dos valores teóricos} em adultos jovens

O valor médio de VT da CVF para homens, calculado por qualquer uma das duas equações analisadas, quando extrapolado para a faixa etária de 17 a 24 é significativamente mais elevado que o encontrado para a faixa de 25 a 30 anos, não sendo as diferenças significativas entre equações para uma mesma faixa etária (Figura 1). Para as mulheres o VT médio calculado por qualquer das equações também foi significativamente mais elevado para a faixa extrapolada (17 a 20 anos) do que para a faixa de 20 a 30 anos. Em ambas as faixas o VT-K foi significativamente maior que o VT-P (Figura 1).

Entre os homens os resultados médios de VT para $\mathrm{VEF}_{1}$ são significativamente mais elevados na faixa extrapolada, o mesmo ocorrendo com as mulheres (Figura 2). Os

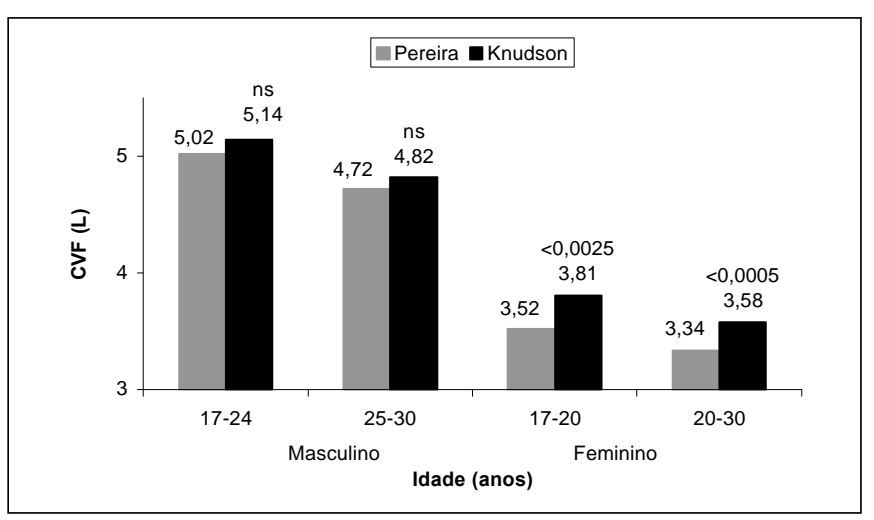

Figura 1 - Comparação entre os valores médios de VT das CVFs obtidas usando as equações de Pereira e Knudson em pacientes adultos jovens de ambos os sexos

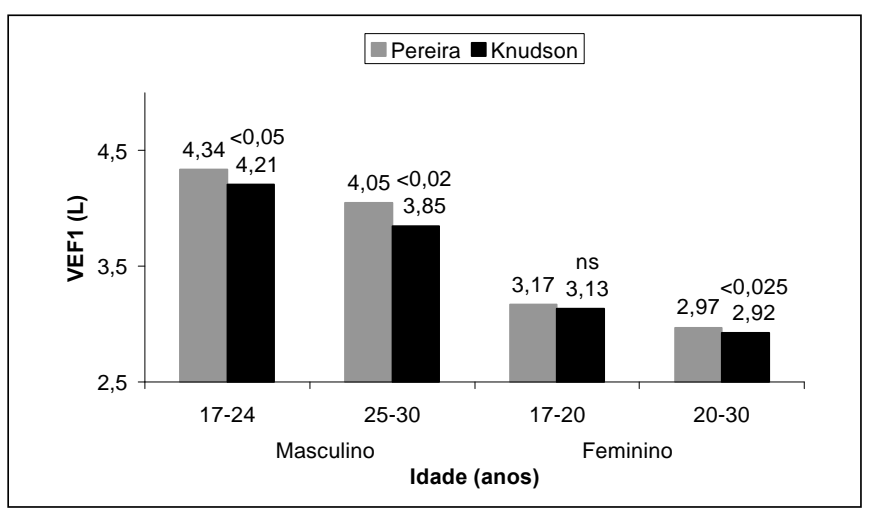

Figura 2 - Comparação entre os valores médios de VT dos VEF, obtidas usando as equações de Pereira e Knudson em pacientes adultos jovens de ambos os sexos 


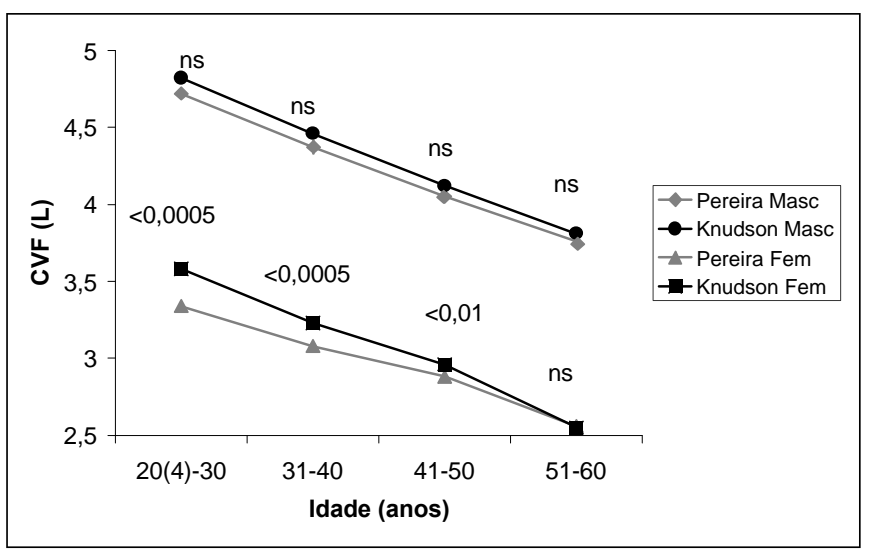

Figura 3 -Variação do VT da CVF, em ambos os sexos, com a progressão da idade

VT-P foram mais elevados que os VT-K e esta diferença foi significativa em todos os grupos, com exceção da faixa extrapolada feminina.

Em virtude do erro introduzido pela extrapolação, serão analisados neste estudo apenas os resultados obtidos em pacientes com idade compreendida dentro dos limites propostos no trabalho de Pereira et al. ${ }^{(25)}$. Desse modo, o universo populacional abordado passou a constituir-se de 344 homens e 660 mulheres.

\section{Diferenças entre as médias dos valores teóricos}

Em adultos do sexo masculino entre 24 e 60 anos, a média de VT-P para CVF foi inferior à média de VT-K $(4,10$ e 4,16 litros, respectivamente). Tal diferença não foi significativa. Já a média dos VT-P para $\mathrm{VEF}_{1}$ foi significativamente maior $(\mathrm{p}<0,005)$ que a média dos VT-K $(3,36$ e 3,20 litros, respectivamente).

Nas mulheres entre 20 e 60 anos, a média dos VT-P para CVF foi significativamente menor $(\mathrm{p}<0,005)$ que a média dos VT-K (2,99 e 3,13 litros, respectivamente). Para $\mathrm{VEF}_{1}$ a média dos VT-P (2,58 litros) foi superior à média dos VT-K (2,47 litros). Tal diferença foi significativa ( $\mathrm{p}<$ $0,005)$.

\section{Variação dos VTs com o avançar da idade}

Analisando-se a evolução dos VTs calculados pelas equações de Pereira e de Knudson, à medida que avança a idade (Figura 3), observa-se que, para o sexo masculino, os valores das médias da CVF permanecem comparáveis $e$ as diferenças não são significativas, qualquer que seja a idade.

Para o sexo feminino, entretanto, os valores obtidos pela equação de Pereira são significativamente mais elevados no grupo entre 20 a 40 anos, diminuindo a diferença com o avançar da idade (grupo entre 41 e 50 anos), desaparecendo para as mulheres acima de 51 anos.

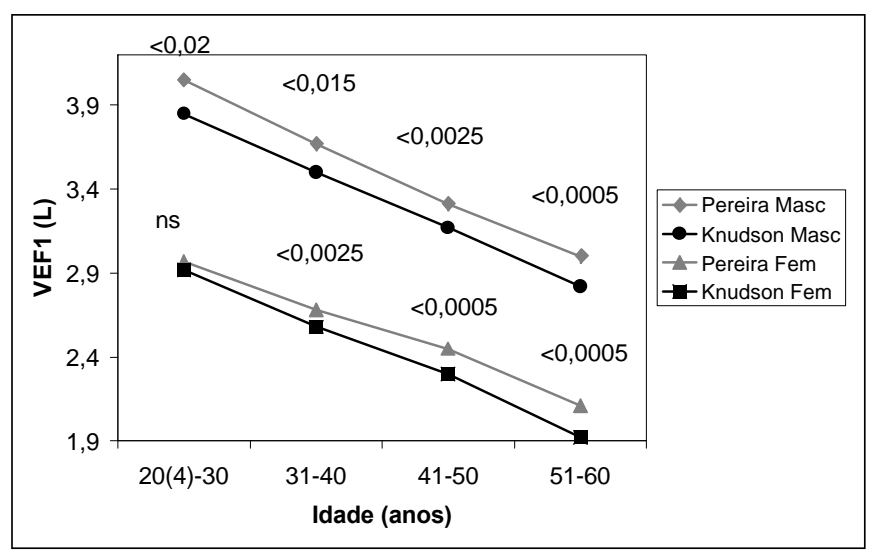

Figura 4 - Variação do VT da $V E F_{1}$, em ambos os sexos, com a progressão da idade

No que concerne ao valor do $\mathrm{VEF}_{1}$, a média dos $\mathrm{VTs}$ encontrados com a equação de Pereira foi, em ambos os sexos, significativamente mais elevada tanto para homens como para mulheres em qualquer das faixas etárias (Figura 4).

Variações de diagnóstico em pacientes masculinos segundo a equação de VT utilizada

Tomando-se por base os diagnósticos feitos com a utilização das equações de Pereira, observa-se que, em 86 pacientes com espirograma dito "normal", a equação de Knudson et al. levou à discordância em sete casos $(8,1 \%)$, todos eles com diagnóstico de dificuldade "restritiva".

Entre os 177 pacientes com diagnóstico de doença "restritiva", houve concordância com a utilização da equação de Knudson ${ }^{(10)}$.

Em 46 pacientes "obstrutivos", a equação de Knudson levou à discordância em 18 (39,1\%). Para esses foram feitos quatro diagnósticos de normalidade, quatro de doença "restritiva" e 10 de insuficiência "mista" ou combinada.

Houve 35 pacientes diagnosticados como "mistos".

Variações de diagnóstico em pacientes femininos segundo a equação de VT utilizada

No grupo feminino, das 217 pacientes diagnosticadas como normais, a equação de Knudson levou em 51 $(23,5 \%)$ ao diagnóstico de restritivo. Entre os 314 "restritivos" a equação de Knudson discordou em apenas um caso $(0,3 \%)$.

Para 81 pacientes com diagnóstico de "obstrutivo", a equação de Knudson discordou em 45 (55,5\%). Para esses firmou oito diagnósticos de normalidade, 14 de doença "restritiva" e 23 de comprometimento "misto".

Houve 48 pacientes com laudo de distúrbio respiratório misto pelas equações de Pereira. 
Evolução das diferenças entre os diagnósticos segundo a idade dos pacientes

Os diagnósticos foram analisados por sexo e em dois grupos etários extremos, adultos jovens (idade entre 25 e 30 anos para homens e de 20 a 30 para mulheres) e adultos maduros (51 a 60 anos para ambos os sexos) (Tabela 1).

Foi observado no grupo mais jovem que, entre os pacientes com diagnóstico de "normalidade", a equação de Knudson levava a discordância em $25 \%$ (três em 12) no sexo masculino e de 34,3\% (24 em 70) no feminino; apresentando-os todos como "restritivos". Essa discrepância desaparece no grupo etário mais velho, no qual somente um de 36 homens $(2,8 \%)$ e nenhuma das 30 mulheres apresentou diagnósticos distintos segundo qualquer das equações utilizadas.

No que concerne ao diagnóstico de defeito "restritivo", somente um paciente em 73 (1,4\%) do grupo mais idoso feminino foi discrepante, enquanto em todos os demais o mesmo foi coincidente com ambas as equações.

No grupo mais jovem, com diagnóstico de insuficiência "obstrutiva", a equação de Knudson levou a discordância em um dentre três homens (33,3\%) e 10 em 16 mulheres $(62,5 \%)$. Devido ao número pequeno da amostra, os resultados não são analisáveis estatisticamente.

Entre os mais velhos, a discrepância é mais acentuada, sendo de $35,3 \%$ (seis de 17) entre os do sexo masculino e de $56,3 \%$ (nove em 16) no sexo feminino.

\section{DisCUSSÃO}

As duas equações comparadas, Pereira e Knudson, ainda que distintas geram para CVF em homens valores que, em uma amostra de população nordestina, não diferiram significativamente. A pequena diferença, entretanto, foi suficiente para que em 86 pacientes considerados "normais" por Pereira $8,1 \%$ fossem identificados como "restritivos" por Knudson.

Uma possibilidade é que o $5^{\circ}$ percentil, medido pela equação de Knudson, se situe num nível um pouco mais elevado que o determinado pela equação de Pereira, levando pacientes com valores de CVF no limite da curva a serem, após comparados com o VT, classificados como "restritivos" por Knudson, mas não por Pereira. Outra possibilidade é que o ligeiro aumento da média do VT calculado por Knudson possa identificar como "restritivos" resultados que são enquadrados como normais por Pereira.

Como a diferença entre as médias do VT para CVF não têm significância estatística, observa-se coerência de diagnóstico de "restritivo" obtido com a aplicação de qualquer uma das duas equações. Na Figura 3 observa-se que a diferença entre as médias permanece não significativa com a evolução da idade e que a concordância em diagnóstico de "restritivo" é observada tanto no grupo mais jovem como no mais idoso.

Entre as mulheres, entretanto, os valores médios encontrados para CVF foram bem diferentes, sendo o de Knudson significativamente mais elevado. Essa diferença se reduz com o aumento da idade, sendo não significativa na faixa etária entre 51 e 60 anos (Figura 3). Como resultado, $23,5 \%$ de 217 pacientes, considerados como "normais", quando calculados por Pereira, foram enquadrados como "restritivos" por Knudson. De 314 classificados como "restritivos" por Pereira, somente um $(0,3 \%)$ não o foi por Knudson.

Analisando por faixa etária (Tabela 1) observa-se que, enquanto houve concordância total no diagnóstico espirométrico de "normalidade" feito em pacientes com mais de 51 anos, houve desacordo de $34,3 \%$ nas pacientes do grupo mais jovem (idade entre 20 e 30 anos), quando a diferença entre valores teóricos é significativa.

A média dos valores teóricos de $\mathrm{VEF}_{1}$ calculados pela equação de Pereira é significativamente mais elevada tanto em homens como em mulheres. Essa diferença permanece estatisticamente significativa em toda faixa etária estudada (Figura 4). Como resultado para um diagnóstico de "obstrutivo" feito com a equação de Pereira, a de Knud-

TABELA 1

Diferenças de diagnóstico segundo a idade dos pacientes

\begin{tabular}{|c|c|c|c|c|c|c|c|c|}
\hline \multirow{3}{*}{$\begin{array}{l}\text { Laudo } \\
\text { Pereira }\end{array}$} & \multicolumn{4}{|c|}{ Masculino } & \multicolumn{4}{|c|}{ Feminino } \\
\hline & \multicolumn{4}{|c|}{25 a 30 anos } & \multicolumn{4}{|c|}{20 a 30 anos } \\
\hline & Pacientes & $\mathbf{K} \neq \mathbf{P}$ & $\%$ & Knudson & Pacientes & $\mathbf{K} \neq \mathbf{P}$ & $\%$ & Knudson \\
\hline Normal & 12 & 3 & 25 & $3 R$ & 70 & 24 & 34,3 & $24 \mathrm{R}$ \\
\hline Restritivo & 22 & 0 & 0 & & 75 & 0 & 0 & \\
\hline \multirow[t]{2}{*}{ Obstrutivo } & 3 & 1 & 33,3 & $1 \mathrm{R}$ & 16 & 10 & 62,5 & $2 \mathrm{~N}, 6 \mathrm{R}, 2 \mathrm{M}$ \\
\hline & \multicolumn{4}{|c|}{51 a 60 anos } & \multicolumn{4}{|c|}{51 a 60 anos } \\
\hline Normal & 36 & 1 & 2,8 & $1 \mathrm{R}$ & 30 & 0 & 0 & \\
\hline Restritivo & 62 & 0 & 0 & & 73 & 1 & 1,4 & $1 \mathrm{Ob}$ \\
\hline Obstrutivo & 17 & 6 & 35,3 & $2 \mathrm{~N}, 1 \mathrm{R}, 3 \mathrm{M}$ & 16 & 9 & 56,3 & $2 \mathrm{~N}, 7 \mathrm{R}$ \\
\hline
\end{tabular}

N - Normal; R - Restritivo; Ob - Obstrutivo; M - Misto. 
son discordou em $39,1 \%$ dos homens e $55,5 \%$ das mulheres.

Para o sexo masculino a discordância em 18 de 46 casos levou a quatro diagnósticos de "normal", mostrando que a de Knudson não identificou valor espirométrico de $\mathrm{VEF}_{1}$ abaixo de seu 5o percentil; quatro foram "restritivos", mostrando que, além de não ter identificado o valor medido abaixo do $5^{\circ}$ percentil do $\mathrm{VEF}_{1}$, ainda enquadrou a CVF abaixo daquele limite. E para 10 pacientes foi estabelecido o diagnóstico de "misto", mostrando que não somente foi identificada por Knudson a insuficiência "obstrutiva" detectada por Pereira, como ainda foi encontrada dificuldade "restritiva".

Para o sexo feminino as mesmas discrepâncias foram encontradas, sendo maior a incidência de diagnóstico de "misto" feito por Knudson em pacientes diagnosticadas como "obstrutivos" por Pereira, provavelmente devido ao valor teórico mais elevado para a CVF.

Concluindo, o presente estudo mostra que para uma amostra aleatória de população brasileira no Nordeste, a utilização da equação de valor teórico de Pereira et al. levou a maior identificação de dificuldade ventilatória obstrutiva que a equação de Knudson. A identificação de dificuldade ventilatória restritiva foi idêntica com o uso de ambas as equações. Em pacientes diagnosticados como "normais" pelo uso da equação de Pereira, o emprego da de Knudson identificou, especialmente em mulheres, um número significativo de dificuldades ventilatórias restritivas.

$\mathrm{Na}$ falta de um "padrão ouro" para definir o diagnóstico correto e tendo-se em conta que as equações brasileiras têm sua raiz em nossa realidade étnica, acreditamos que essas sejam as mais precisas para o uso corrente em nosso meio.

\section{REFERÊNCIAS}

1. Burrows B, Lebowitz MD, Camili AE, Knudson RJ. Longitudinal changes in forced expiratory volume in one second in adults. Am Rev Respir Dis 1966;133:974-980.

2. Cotes JE. Lung function throughout life; determinants and references values. In: Lung function. Assessment and application in medicine. $2^{\text {nd }}$ ed. Oxford: Blackwell Scientific Publications, 1979;329-388.

3. Schwartz J, Katz SA, Fegley RW, Tockman MS. Sex and race differences in the development of lung function. Am Rev Respir Dis 1988; 138:1415-1421.

4. Knudson RJ, Slatin RC, Lebowitz MD, Burrows B. The maximal expiratory flow-volume curves. Normal standards variability and effect of age. Am Rev Respir Dis 1976;113:587-600.

5. Higgins M, Keller JB, Wagenknecht LE, et al. Pulmonary function and cardiovascular risk factor relationship in black and in white men and women. Chest 1991;99:315-322.

6. Rossiter CE, Weil H. Ethnic differences in lung function. Evidence for proportional differences. Int J Epidemiol 1974;3:55-61.

7. Schonberg JB, Beck GT, Bouhuys A. Growth and decay of pulmonary function in healthy blacks and whites. Respir Physiol 1978;33:367-393.

8. Korotzer B, Ong S, Hansen JE. Ethnic differences in pulmonary function in healthy nonsmoking Asian-Americans and European-Americans. Am J Respir Crit Care Med 2000;161:1101-1108.
9. Ashley F, Kannel WB, Sorlie PD, Masson R. Pulmonary function: relation to aging, cigarette habit and mortality. The Framington study. Ann Inter Med 1975;82:739-745.

10. Knudson RJ, Slatin RC, Lebowitz MD, Burrows B. The maximal expiratory flow-volume curves. Normal standards variability and effect of age. Am Rev Respir Dis 1976;113:587-600.

11. Knudson RJ. How aging affects the normal adult lung. J Respir Dis 1981;2:74-84.

12. Knudson RJ, Lebowitz MD, Holberg CJ, Burrows B. Changes in the maximal expiratory flow-volume curve with growth and ageing. Am Rev Respir Dis 1983;127:725-734.

13. Glindmeyer HW, Diem JE, Jones RN, Weill H. Noncomparability of longitudinally and cross-sectionally determined annual change in spirometry. Am Rev Respir Dis 1982;125:544-548.

14. Hutchinson J. On the capacity of the lungs and on the respiratory function with a view of establishing a precise and easy method of detecting disease. Med Chir Soc Trans 1986;29:137-252.

15. Dybaker R, Grasbeck R. Theory of reference value. Scand J Lab Invest 1973;32:17.

16. Buist AS. Evaluation of lung function: concepts of normality. Curr Pneumol 1983;4:141-165.

17. Crapo RO, Morris AH, Gardner RM. Reference spirometric values using techniques and equipment that meets the ATS recommendations. Am Rev Respir Dis 1981;123:659-664.

18. Morris JF, Koski A, Johnson LC. Spirometric standards for healthy nonsmoking adults. Am Rev Respir Dis 1971;103:67-167.

19. Quanjer Ph H, Tammeling G, Cotes JE, Pedersen R, Peslin R, Yernault J-C. Lung volumes and forced ventilatory flows. Report working party standardization of lung function tests. European Community for Steel and Coal. Official statement of the European Respiratory Society. Eur Respir J 1993;(Suppl)16:5-40.

20. Sedlaczek AM, Rulkowska H. Comparative analysis of basic spirometric parameters recognized by different authors as normal values for the same individuals. Pneumol Pol 1989;10-12:471-476.

21. Rulkowska H. Comparative analysis of basic spirometric parameters accepted by various authors as values (norms) belonging to the same person, and an attempt to find values of these parameters that are proper for the population of young males in Poland. Ann Acad Med Stetin 1994;40:79-95.

22. Gore CJ, Crockett AJ, Pederson DG, Booth ML, Bauman A, Owen N. Spirometric standards for healthy adult lifetime nonsmokers. Eur Respir J 1995;8:773-782.

23. ATS. Lung function testing: selection of reference values and interpretation. Am Rev Respir Dis 1991;144:1202-1218.

24. Romaldini H. Quando vamos parar de adaptar padronizações pneumológicas? J Pneumol 1978;4:16-26.

25. Pereira CAC, Barreto SP, Simões JG, et al. Valores de referência para espirometria em uma amostra da população brasileira. J Pneumol 1992; 18:10-12.

26. Dickman ML, Schmidt CD, Gardner RM. Spirometric standards for normal children and adolescents (ages 5 years through 18 years). Am Rev Respir Dis 1973;108:933-939.

27. Seely JE, Guzman CA, Beclake MR. Heart and lung function at rest and during exercise in adolescence. J Appl Physiol 1974;36:34-40.

28. Knudson RJ, Lebowitz MD, Holberg CJ, Burrows B. Changes in the normal maximal expiratory flow-volume curve with growth and aging. Am Rev Respir Dis 1983;127:725-734.

29. Mallozi MC. Valores de referência para espirometria em crianças e adolescentes, calculados a partir de uma amostra da cidade de São Paulo [Tese]. São Paulo: Escola Paulista de Medicina, 1995;116p.

30. SBPT I Consenso Brasileiro de Espirometria. J Pneumol 1966;22: 105-156.

31. D'Angelo E, Prandi E, Marazzini L, Milic-Emili J. Dependence of maximal flow-volume curves on time course of preceding inspiration in patients with chronic obstructive pulmonary disease. Am J Respir Crit Care Med 1994;150:1581-1586. 